\title{
Is the Wireless Monitoring a Technological Innovation Suitable in Internal Medicine Units?
}

\author{
Pietrantonio $\mathrm{F}^{*}$ \\ Internal Medicine Unit, Castelli Hospital, ASL Roma6, Italy \\ *Corresponding author: Pietrantonio F, Internal Medicine Unit, Castelli Hospital, ASL Roma6, Italy
}

Submission: February 04, 2019; Published: February 07, 2019

\section{Editorial}

In recent years, the Internal Medicine (IM) Ward has been receiving an increasingly heterogeneous group of patients, many affected by acute or chronic diseases of varying severity, who are often elderly, frail, with co-morbidities, requiring intensive care due to epidemiological transition. Hospitalization of medical patients in large wards without prior stratification of severity, complexity, level of dependence, co-morbidities and without a proper assessment of the risk of rapid clinical deterioration, can lead to suboptimal treatment, resulting in prolonged hospital stay and increased care costs. Continuous monitoring of Emergency Room (ER) activity data has shown an increasing trend, regardless of the season, in ER presentation and subsequent hospitalization of patients over 65 years with exacerbation of chronic diseases such as a $1 \%$ increase in ER presentations could result in a $10 \%$ increase in Internal Medicine admissions, causing overcrowding both of the ER and IM ward [1].

A recent retrospective study in the IM Department demonstrated that $27 \%$ of patients admitted to the IM ward are critical (needing continuous monitoring and advanced medical technology), 10\% of which fulfilled criteria for intensive care transfer: MEWS $\geq$ 5) presenting 5-6 active pathologies. Furthermore, 25\% of hospitalized patients had social difficulties requiring activation of integrated hospital-territory pathways, to ensure appropriate care, it is therefore essential to stratify patients based on clinical intensity and care complexity and to define end-stage disease in order to provide adequate nursing settings, staff standards and the necessary technological equipment [2]. In order to provide data on the clinical impact of acute and critically ill patients in an Internal Medicine Unit, as well as economic data regarding the relative cost of acute patient management during ordinary hospitalization, and to assess whether continuous monitoring of vital parameters improves quality of care, a randomized multicenter study is underway, proposed by the Internal Medicine Unit of Manerbio Hospital (ASST-Garda) in collaboration with the University of Modena and Reggio Emilia (Medical Statistics Unit), Internal Medicine Unit of Castelli Hospital, ASL Roma 6 and Internal Medicine Unit 1 of Villa Sofia Hospital in Palermo [3]. The study aims at assessing the efficacy of a wireless continuous monitoring system in reducing major complications (primary end-point) and improving outcome and the quality of care (secondary end-points) in acute selected patients, while reducing costs, as compared to traditional monitoring performed at regular intervals by the nursing staff. Continuous monitoring of vital signs may permit detection of deterioration of acute patients in a non-intensive care setting (such as those hospitalized in the Internal Medicine Unit), allowing staff to promptly respond urgently to variations in the patient's condition and initiate appropriate care [4]. In order to respond to an apparent lack of specific studies using scores to quickly determine the degree of illness of a patient, based on the vital signs (such us MEWS-Modified Early Warning Score [5] and NEWS- National Early Warning Score) and comparing the use of wireless monitoring systems and traditional vital sign monitoring in critical acute patients, the study was designed to highlight the benefits of continuous monitoring of vital signs in the first 72 hours of hospitalization [6,7]. The study also indirectly aims to assess the reduction in hospitalization costs by using the decrease in average length of stay (LOS) as a proxy parameter. The wireless monitor is realized by a simple device, WIN @ Hospital system (Medical Class IIA), a Medical Class II light monitoring system trade registered with the code A CE0434 in accordance with Directive 93/42/EEC. Win@Hospital monitoring systems allow real-time data display, but do not require the continuous presence of nursing staff monitoring data, because, as a class IIA device, it is not designated as an intensive monitoring tool nor life-saving (class II B), but a simple support tool aimed at tracking clinical parameters (to improve diagnosis, for the automation of detection procedures and for the reduction of clinical risk).

Very interesting are the preliminary results [8] that confirms the validity of the innovation applied to improve the management of patients admitted to Internal Medicine Units. During the first year, 100 patients were recruited and 89 evaluated (35M/54F), with a mean age 80.5 years, mean CIRS-CI of 4 , and CIRS SI of 1.8. Main DRGs (Diagnosis Related Groups): 127 (heart failure), 87 (respiratory failure), 576 (sepsis). End-stage disease so far represents more than $30 \%$ of the sample of the 89 patients, $38 \%$ had a BRASS score of $\geq 20$ indicating cases where discharge may 
present difficulties and planning of step-down care is required. More than $50 \%$ of patients presented with an IIA index $>3$ indicating high dependence on nursing assistance. Nurses saved a minimum of 49,6 minutes to a maximum of 58, 1 minutes on time spent monitoring each patient per day, which may translate to increased time spent on other activities which contribute to patient wellbeing Of a total 1998 patients admitted in one year (2017 activity data of Manerbio Internal Medicine), only 5\% were recruited out of the $27 \%$ deemed eligible, due to conditions such as dementia, coma or end stage disease which rendered signing of the informed consent impossible. The preliminary results are encouraging in that a trend towards reduction of major complications in the experimental group appears to be seen (31\% in experimental arm versus $45 \%$ in the control arm). Moreover, a decrease in re-admissions (7\% versus $11 \%$ ) and, impressively, mortality (7.3\% versus $23.9 \%$ ) has been observed. More than $30 \%$ of the patients meet the criteria defining end stage disease (positive answer to "surprise question": "Would I be surprised if this patient died in the next 12 months?"); $42.5 \%$ in the experimental arm and $33.3 \%$ in the control arm.

Preliminary results have failed to confirm the hypothesis regarding reduction in hospital stay, showing, on the contrary, increased LOS in the experimental arm (11.5 days versus 10.6 days), This result is probably due to the evidence that in the experimental arm the BRASS $>20$ (need for discharge planning) is higher $333.3 \%$ versus $22.2 \%$ ). Contrary to current beliefs, upheld nonetheless by scientific studies, the hospital internist regularly faces complex, unstable patients with difficult diagnoses and multiple comorbidities, who pass into their care once relatively stabilized. Management of this phase requires the specific competencies of the internist in diagnosis and management of the unstable, acutely unwell patient. However, the burden of management of clinical complexity, comorbidities, frailty, disability and social problems is appropriately shared with other disciplines (e.g. geriatricians, physiatrists) and may occur in the setting of assisted-care residential facilities, long-term care, home healthcare, or in collaboration with General Practitioners etc. The increasing role of Internal Medicine units in the management of urgent admissions can be partially explained by the fact that an IM ward is generally present in all hospitals and usually boasts a higher number of beds compared to specialized wards (Geriatrics, Neurology, Pediatrics, etc.), which are less well represented and have a lower number of beds [9]. According to the Literature [10], Internal Medicine units admit mainly complex DRGs with neurological or cardiac issues as well as many patients fitting criteria for Geriatric Unit admission. Older age, together with polypharmacy, absence of formal and/or informal home-help services, history of falls, temporal disorientation, type of residence and use of psychoactive drugs significantly increase likelihood of hospital admission through the ER.

Considering that $55 \%$ of hospital admissions in Italy are urgent, and that the IM unit receives $27 \%$ of the total emergency admissions, not to mention that $83 \%$ of the IM admissions come from the ER, the prevalent activity in IM units is characterized by management of the acute complex patient[11].
ACUTE COMPLEX CARE MODEL: [12] In consideration of this data, the Acute Complex Care Model (ACCM) appears an appropriate strategy management for acute patients presenting to the ER and admitted to Internal Medicine wards. The ACCM represents the hospital-based counterpart of the Chronic Care Model (CCM) and is similarly aimed to ensure effective care, often requiring the support of advanced technology, of complex and poly-pathological patients (CIPs) when they are hospitalized for acute diseases. The mission is to improve management of medical admissions by establishing pre-defined intra-hospital pathways and a global, multidisciplinary, patient-centered approach. The strengths of the ACCM are represented by:

A. In-hospital care pathways, based on patients' needs and tailored towards CIPs needs

B. Ability to balance risk and benefits of specialist treatments in any single patient

C. Managerial competencies applied to CIPs and multiprofessional integration.

The acute poly-pathological patient should be identified immediately upon arrival at the hospital and the need for hospitalization should be carefully evaluated, as should alternative outpatient options. Considered selection of patients for admission to the hospital is essential to avoid overcrowding of emergency and medical wards, to reduce clinical risk, and to allow appropriate and timely care. A patient with a single-organ acute disease, but with multiple chronic comorbidities and in tenuously stable clinical condition, should be immediately taken into the care of an internist. This ensures a more holistic approach, as the internist, by virtue of their specific training and competencies, represents the ideal medical figure not only to coordinate specialist physician consultations as appropriate, but carefully balance management of comorbidities and the accompanying issue of polypharmacy based on the patient's current health status.

A clinical summary and a list of priorities should be written down immediately after admission, and the composition of the multidisciplinary team, required by patient's health conditions, should be clearly reported in the medical records. According to validated criteria, for example the MEWS and the NEWS scores, vital parameters should be monitored continuously or at regular intervals, and wireless systems may permit creation of an adequate care setting for the patient without having to move them to a different department. Continuous monitoring of vital parameters may allow early detection of deterioration in acute patients not admitted to an intensive care unit such as those present on the Internal Medicine Unit, allowing the staff to immediately address the patient's needs and promptly initiate the most appropriate care. As there are no studies comparing the use of wireless monitoring systems and traditional monitoring of vital signs in critical acute patients, the study would like to help highlight the benefits of continuous monitoring of vital signs during hospitalization in order to reduce major complications, to improve outcomes and to reduce 
costs. The preliminary results are encouraging as they appear to highlight a trend towards continuous monitoring over traditional monitoring in terms of reduction in major complications.

Continuous wireless monitoring of vital parameters may permit early detection of deterioration in critically ill patients allowing prompt intervention, increasing safety and reducing costs. Extending the wireless monitoring system to low intensity facilities and indeed to the home setting may guarantee expert assistance following hospital discharge, reducing overcrowding in the ER and IM units. Doing the right thing to the right patient at the right time represents the core mantra guiding quality diagnostic-therapeutic pathways that, accompanied by appropriate and efficient clinical reasoning, is the only way to reduce costs and length of hospital stay while at the same time delivering care at a level of excellence [13]. An important role of the Internal Medicine Unit is improving clinical conditions of patients otherwise in the terminal phase of their illness, such that life expectancy is indeed prolonged. Therefore, the Internal Medicine Unit also contributes to the active management of end-stage patients, as need for palliative care is effectively deferred as this newfound clinical stability actually allows discharge after the acute phase.

\section{References}

1. Pietrantonio F, Scotti E (2017) Internal medicine network: a new way of thinking hospital-territory integration and public-private partnership. Italian Journal of Medicine 11(2): 85-94.

2. Pietrantonio F, Aperti P, Tonoli L, Tyndall E, Meneghetti O (2018) The correct setting to improve the quality of health care process: a retrospective study in Internal Medicine Department. It J Med 12(4): 285-295.

3. Fueglistaler P, Adamina M, Guller U (2007) Non-inferiority trials in surgical oncology. Ann Surg Oncol 14(5): 1532-1539.
4. Nardi R, Scanelli G, Borioni D, Grandi M, Sacchetti C, et al. (2007) The assessment of complexity in internal medicine patients. The FADOI Medicomplex Study. Eur J Int Med 18(4): 283-287.

5. Subbe CP, Kruger M, Rutherford P, Gemmel L (2001) Validation of a modified early warning score in medical admissions. Q J Med 94(10): 521-526.

6. Scotti E, Pietrantonio F (2013) The hospital internal medicine specialist today: a literature review and strength, weaknesses, opportunity, threats (SWOT) analysis to develop a working proposal. It J Med 7(4): 278-286.

7. Launay CP, Rivière H, Kabeshova A, Beauchet $O$ (2015) Predicting prolonged length of hospital stay in older emergency department users: Use of a novel analysis method, the artificial neural network. Eur J Intern Med 26(7): 478-482.

8. Pietrantonio F, Bussi AR, Amadasi S, Bresciani E, Caldonazzo A Colombini P, Giovannini MS, Grifi G, Lanzini L, Migliorati P, Perini P, et al. Technological Challenges set up by Continuous Wireless Monitoring designed to Improve Management of Critically Ill Patients in an Internal Medicine Unit (LIMS study): Study Design and Preliminary Results. J Community Prev Med 2019; 2(1):1-7.

9. Brown H, Terrence J, Vasquez P, Bates DW, Zimlichman E (2014) Continuous monitoring in an in-patient medical-surgical unit: a controlled clinical trial. Am J of Medicine 127(3): 226-232.

10. Launay CP, Rivière H, Kabeshova A, Beauchet O. Predicting prolonged length of hospital stay in older emergency department users: Use of a novel analysis method, the artificial neural network. Eur J Intern Med 2015;26:478-82.

11. Pietrantonio F, Piasini L, Spandonaro F (2016) Internal medicine and emergency admissions: from a national hospital discharge records (SDO) study to a regional analysis. It J Med 10(2): 157-167.

12. Pietrantonio F, Orlandini F, Moriconi L, La Regina M (2015) Acute complex care model: An organizational approach for the medical care of hospitalized acute complex patients. Eur J Intern Med 26(10): 759-765.

13. Mongardi M, Bassi E, Ruscio ED (2015) Ospedale per Intensità di cura: strumenti per la classificazione dei pazienti. DGSPS, Servizio Presidi Ospedalieri, Regione Emilia-Romagna, Italy.

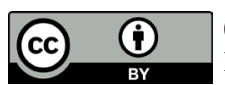

Creative Commons Attribution 4.0 International License

For possible submissions Click Here

\section{Submit Article}

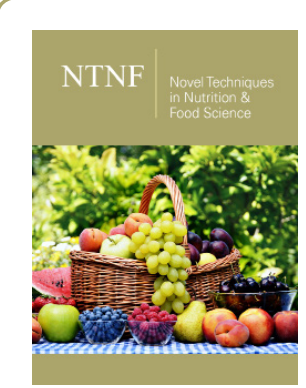

\section{Novel Techniques in Nutrition and Food Science}

\section{Benefits of Publishing with us}

- High-level peer review and editorial services

- Freely accessible online immediately upon publication

- Authors retain the copyright to their work

- Licensing it under a Creative Commons license

- Visibility through different online platforms 